\title{
Computing light pollution indicators for environmental assessment
}

\author{
Fabio Falchi ${ }^{1}$ and Salvador Bará ${ }^{1}$ \\ ${ }^{1}$ Universidade de Santiago de Compostela
}

April 6, 2021

\begin{abstract}
Light pollution modelling and monitoring has traditionally used zenith sky brightness as its main indicator. Several other indicators (e.g. average sky radiance, horizontal irradiance, average sky radiance at given interval of zenith distances) may be more useful, both for general and for specific purposes of ecology studies, night sky and environmental monitoring. These indicators can be calculated after the whole sky radiance is known with sufficient angular detail. This means, for each site, to integrate the contribution in each direction of the sky of each light source in the radius of hundreds of km. This approach is extremely high time consuming if the mapping is desired for a large territory. Here we present a way to obtain maps of large territories for a large subset of useful indicators, bypassing the need to calculate first the radiance map of the whole sky in each site to obtain from it the desired indicator in that site. For each indicator, a point spread function (PSF) is calculated from the whole sky radiance maps generated by a single source at sufficiently dense number of distances from the observing site. If the PSF is transversally shift-invariant, i.e. if it depends only on the relative position of source and observer, then we can further speed up the map calculation via the use of fast Fourier-transform (FFT). We present here examples of maps for different indicators. Precise results can be calculated for any single site, taking into account the site and light sources altitudes, by means of specific inhomogeneous (spatially-variant) and anisotropic (non rotationally symmetric) PSFs.
\end{abstract}

\section{Hosted file}

Computing light pollution indicators for environmental assessment.pdf available at https://authorea.com/users/10622/articles/516881-computing-light-pollution-indicatorsfor-environmental-assessment 